\title{
TRATAMIENTO ENDOUROLÓGICO PERCUTÁNEO DE LITIASIS URETERAL OBSTRUCTIVA EN INJERTO RENAL
}

\author{
F. RAMÓN DE FATA CHILLÓN, C. NÚÑEZ MORA, J.Ma. GARCÍA MEDIERO, \\ J.M ${ }^{a}$. ALONSO DORREGO, L. HIDALGO TOGORES, J.J. DE LA PEÑA BARTHEL
}

Unidad de Litiasis y Endourología. Servicio de Urología. Hospital Universitario La Paz. Facultad de Medicina. Universidad Autónoma de Madrid. Madrid.

Actas Urol Esp. 27 (1): 39-42, 2003

\section{RESUMEN \\ “TRATAMIENTO ENDOUROLÓGICO PERCUTÁNEO DE LITIASIS URETERAL OBSTRUCTIVA EN INJERTO RENAL"}

En el riñón trasplantado la aparición de litiasis representa una de las complicaciones menos frecuentes, sin embargo, dadas las características de estos pacientes, se asocia a una elevada morbilidad. Varias son las causas responsables de esta enfermedad: se han identificado factores metabólicos, infecciosos, fármacos, cuerpos extraños, así como la presencia previa de litiasis en el propio injerto. Desde el punto de vista del tratamiento, dado que son pacientes con un riñón único funcionante, la agresión a que éste sea sometido debe ser lo menor posible. Presentamos un caso de litiasis ureteral obstructiva de gran dureza, en un injerto renal, resuelta mediante acceso percutáneo anterógrado con ureteroscopio flexible, con éxito.

PALABRAS CLAVE: Litiasis. Trasplante renal. Tratamiento.

\section{ABSTRACT \\ “ENDOUROLOGICAL MANAGEMENT OF URETERAL OBSTRUCTIVE CALCULI AFTER RENAL TRANSPLANTATION"}

Donor graft lithiasis is a unusual complication of renal transplantation, however, it is associated to a high morbidity. This pathology is due to several causes such us: metabolic factors, infectious disease, drugs, foreign bodies or transferred in the donor graft. The objetive of the treatment is to remove the lithiasis without damaging the renal unit. We report the succesful percutaneous anterograde treatment of an ureteral obstructive hard calculi, in renal allograft.

KEY WORDS: Lithiasis. Renal transplantation. Treatment.

$\mathrm{L}$ a litiasis urinaria es una complicación rara del trasplante renal, con una incidencia global del 1-2\%1. Su etiología es multifactorial y entre los factores litogenéticos implicados se han señalado: la infección urinaria recurrente, la obstrucción del tracto urinario, la presencia de cuerpos extraños en contacto con la orina que actuarían como matriz del cálculo (suturas irreabsorbibles, catéteres), factores metabólicos, fármacos como la ciclosporina A (uricosuria) o la transferencia con el propio injerto. La composición de los cálculos no difiere de los observados en pacientes no trasplantados. Se han utilizado distintos procedimientos terapéuticos para su resolución, debiendo utilizar siempre el menos invasivo, ante la posibilidad de recurrencia y para preservar la función del injerto, siendo el manejo endourológico una alternativa terapéutica en aquellos casos refractarios a litotricia extracorpórea por ondas de choque ${ }^{1}$.

\section{CASO CLÍNICO}

Varón de 53 años con antecedentes personales de hipertensión arterial en tratamiento con 
nifedipino, talasemia minor, accidente cerebrovascular en territorio carotídeo derecho en tratamiento con antiagregantes y trasplante renal de donante cadáver, por insuficiencia renal crónica secundaria a hialinosis focal y segmentaria hace 5 años, realizado en otro centro, en tratamiento inmunosupresor con azatioprina, ciclosporina y prednisona.

El paciente presentó dolor agudo en fosa ilíaca derecha y disminución de la diuresis por lo que acudió a su centro de referencia, siendo diagnosticado de litiasis radiopaca de $7 \mathrm{~mm}$, de diámetro máximo, en el uréter yuxtavesical del injerto renal, causante de uropatía obstructiva, con empeoramiento de la función renal (creatinina: $2,1 \mathrm{mg} / \mathrm{dl}$ ), que requirió derivación urinaria (nefrostomía percutánea derecha).

$\mathrm{El}$ paciente fue remitido a nuestro Servicio para tratamiento. En la radiografía simple de aparato urinario se apreciaba una imagen de densidad calcio de $7 \times 5 \mathrm{~mm}$. en el uréter distal del injerto, clips metálicos en fosa ilíaca derecha en relación con la cirugía previa y catéter de nefrostomía. La ureteropielografía anterógrada por nefrostomía demostró una litiasis menor de $1 \mathrm{~cm}$. de diámetro máximo, de alta densidad radiológica, parcialmente obstructiva, con paso distal de contraste (Fig. 1). La función renal se había normalizado (creatinina: $1,3 \mathrm{mg} / \mathrm{dl}$, aclaramiento de creatinina: $107 \mathrm{ml} / \mathrm{min}$.), el estudio de coagulación era normal y el urocultivo estéril.

Se realizó litotricia extracorpórea por ondas de choque en régimen ambulante, aplicándose 3.500 choques con una energía total de 1.200 Julios sin evidenciarse fragmentación del cálculo durante el procedimiento, ni a los 2 meses.

Se decidió proceder a tratamiento endourológico de la litiasis. Bajo anestesia general y en posición de litotomía se intentó realizar acceso endoscópico retrógrado del uréter reimplantado, siendo esto imposible por encontrarse la ureteroneocistostomía en fondo vesical, en zona móvil, lo que impidió su cateterización. En el mismo acto, se procedió a realizar una dilatación progresiva del trayecto percutáneo preexistente (nefrostomía $8 \mathrm{Ch}$.), hasta lograr introducir vaina de Amplatz $15 \mathrm{Ch}$., lo que posibilitó la nefroureteroscopia anterógrada, con ureteroscopio flexible (Olympus $10 \mathrm{Ch}$.), del injerto renal. La litiasis fue fragmentada mediante litotricia electrohidráulica con sonda flexible bajo visión directa y los fragmentos litiásicos fueron extraídos con

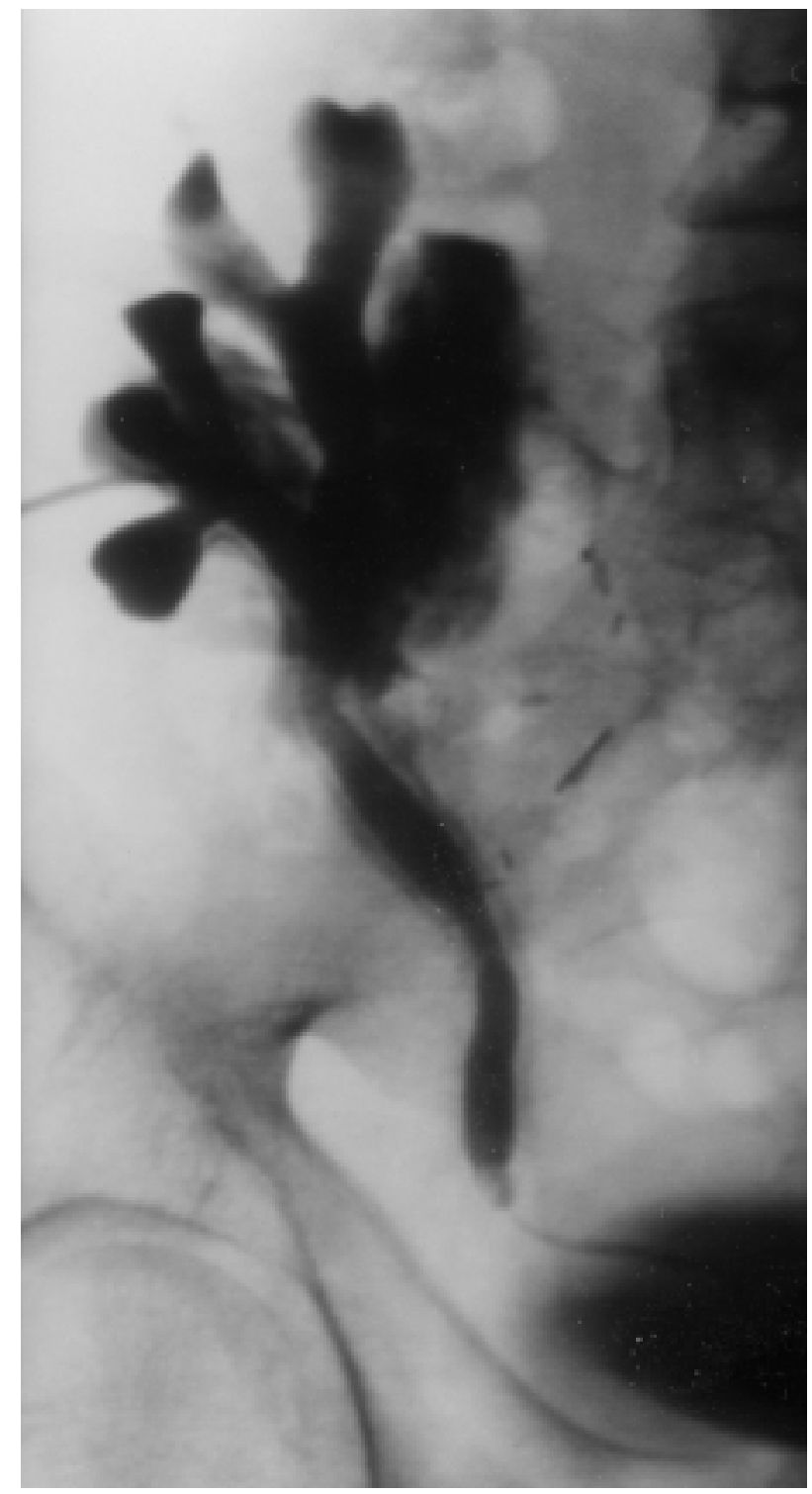

FIGURA 1. Pielografia anterógrada por nefrostomia.

pinza de cuerpo extraño. Se colocó anterógradamente un catéter ureteral pig-tail de calibre $7 \mathrm{Ch}$. y se recambió el catéter de nefrostomía.

A las 24 horas de la intervención quirúrgica, se realizó una pielografía anterógrada por el catéter de nefrostomía, confirmándose la ausencia de fragmentos litiásicos y con buen paso de contraste a vejiga, por lo que se retiró el catéter de nefrostomía.

El paciente fue dado de alta a las 48 horas, sin complicaciones. En la urografía intravenosa de control a las 4 semanas de la intervención se demostró la ausencia de litiasis en la vía excretora del injerto (Fig. 2), siendo retirado el catéter ureteral mediante cistoscopia ambulante. 


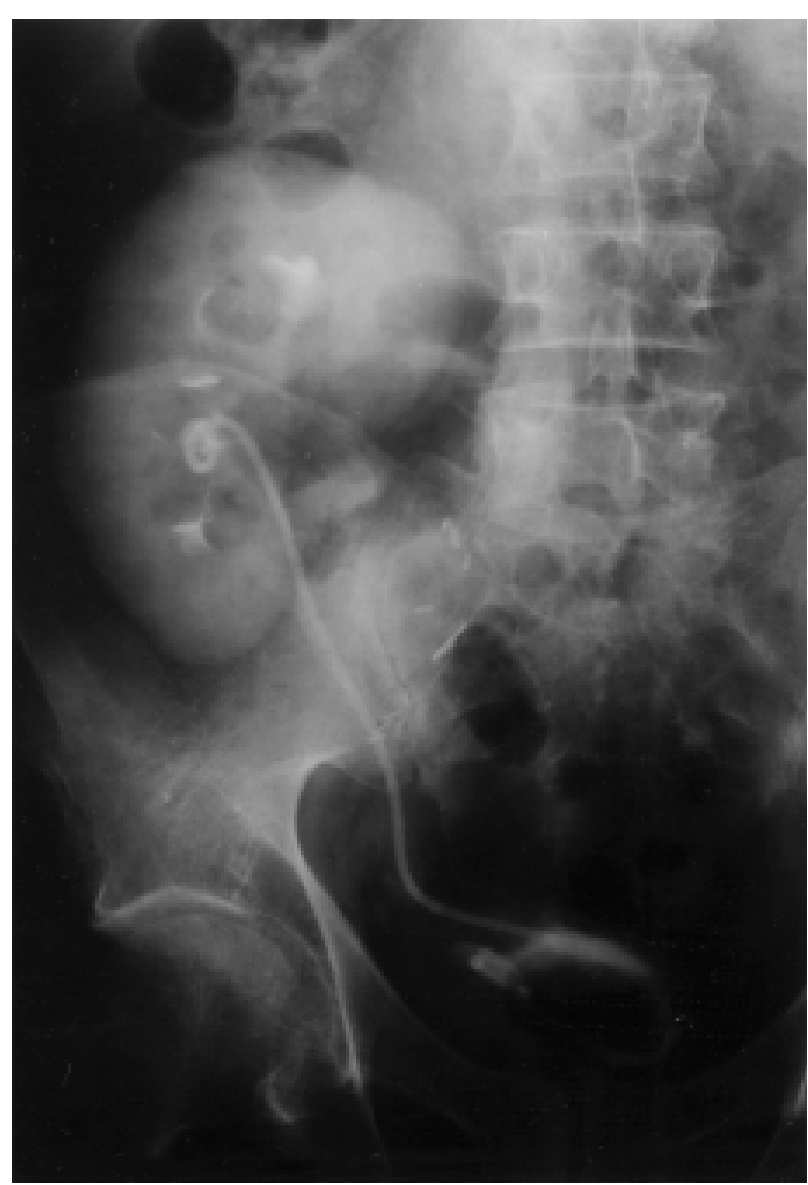

FIGURA 2. Urografia intravenosa postoperatoria.

El análisis del cálculo fue de oxalato cálcico monohidrato.

El paciente se encuentra asintomático, sin litiasis y con una función renal conservada 3 meses después del procedimiento.

\section{DISCUSIÓN}

La litiasis es una de las complicaciones menos frecuentes del trasplante renal, con una incidencia del 1-2\%, habiéndose identificado hasta la fecha alrededor de 200 casos en la literatura $^{1}$, pero con una elevada morbilidad, al tratarse de pacientes con un riñón único funcionante asociado a inmunosupresión.

La etiopatogenia de la urolitiasis en el riñón trasplantado es multifactorial ${ }^{2}$. Entre los factores litogénicos identificados, destacan las anomalías metabólicas, siendo la hipercalcemia el factor litogénico principal en algunas series ${ }^{3}$, la hiperuricemia con hiperuricosuria aparece hasta en el 50\%, según otros autores ${ }^{4}$, el hiperparatiroidismo

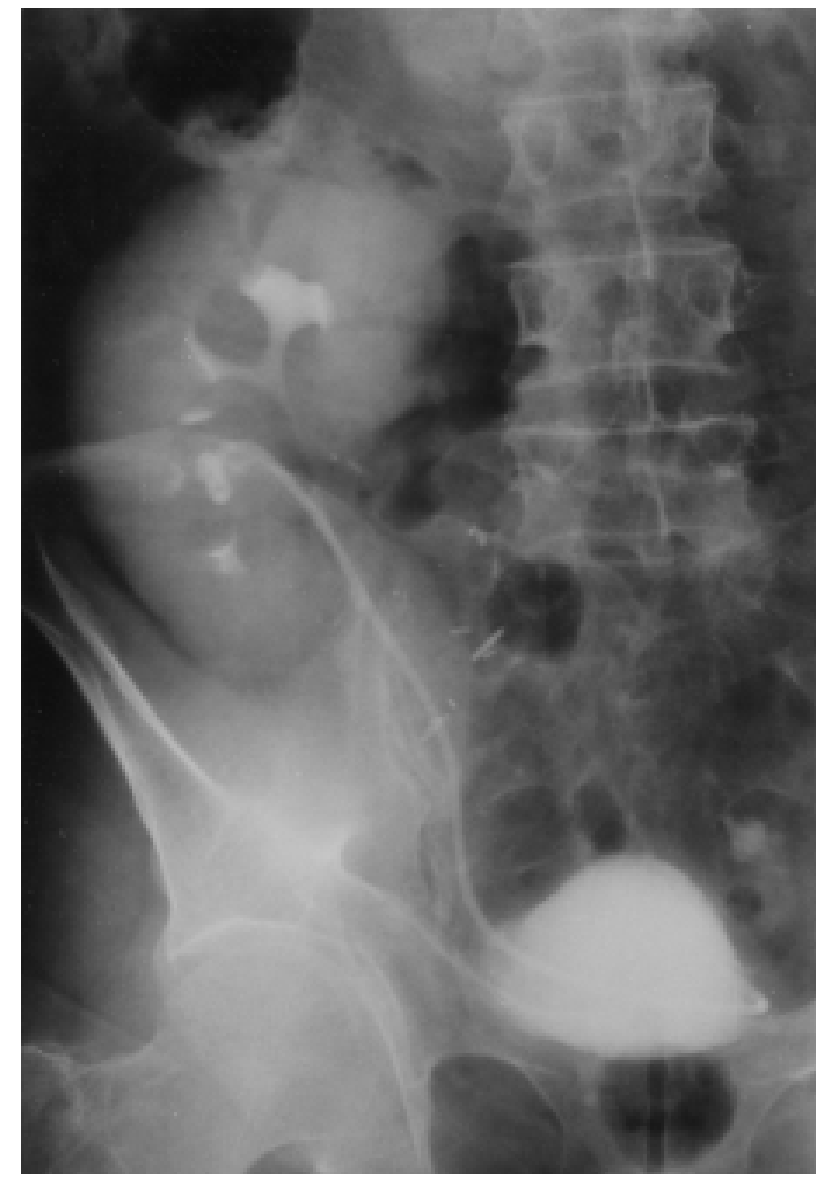

secundario, la acidosis tubular renal, la hiperoxaluria y la disminución en la excreción urinaria de citrato son otros de los factores señalados ${ }^{1,2}$.

Cabe destacar que el tratamiento inmunosupresor con ciclosporina A aumenta la uricemia y la uricosuria y acidifica la orina, lo que contribuye a la formación de cálculos de ácido úrico.

Entre los factores litogénicos locales destacan las infecciones urinarias de repetición, el uso de conductos ileales, la obstrucción del tracto urinario o la presencia de cuerpos extraños, como catéteres o suturas irreabsorbibles, utilizadas hace años en la realización de la anastomosis ureterovesical y que producen una nucleación heterogénea. Montayne y cols. presentan una incidencia de litiasis postrasplante superior al 6\%, en relación con el uso de grapas metálicas para realizar la ureteroneocistostomía ${ }^{5}$. Esta práctica ha sido abandonada hoy en día al lograr idéntica estanqueidad con suturas reabsorbibles, disminuyendo la formación de cálculos. 
También ha sido descrita la transferencia de litiasis con el injerto, cuyo primer caso fue publicado por Lerut y cols. en $1979^{6}$.

En cuanto a la presentación clínica, es rara la aparición de un dolor cólico típico secundario a la dilatación de la vía excretora, debido a la denervación del uréter del injerto. El inicio de oligoanuria, infección urinaria de repetición, hematuria o deterioro de la función renal en el análisis de sangre, nos debe hacer descartar la existencia de litiasis en el riñón trasplantado ${ }^{2,4}$.

El tratamiento de la litiasis en el injerto renal depende de las características del cálculo, de la vía urinaria y de los síntomas del paciente. La observación y vigilancia periódica está indicada en los pacientes asintomáticos, con función renal normal, urocultivo estéril y en litiasis de pequeño tamaño (menor de $4 \mathrm{~mm}$.), situadas en cálices renales, sin repercusión sobre la vía excretora, o en uréter yuxtavesical ${ }^{1}$; aunque en éste último caso es necesario extremar la vigilancia al tratarse de pacientes monorrenos e inmunodeprimidos.

En los pacientes con litiasis radiotransparentes, sugestivas de estar compuestas de ácido úrico, se debe intentar una litolísis química con fármacos alcalinizantes (citrato potásico, bicarbonato sódico) e hidratación ${ }^{1,4}$, aunque se debe tener precaución con los niveles de potasio sérico, dado que se trata de pacientes monorrenos y muchos de ellos con hipertensión arterial y en tratamiento con diuréticos. Asimismo la sobrecarga de sodio inducida por el bicarbonato sódico puede agravar la posible hipertensión arterial de los pacientes, ya sea esencial o secundaria a fármacos inmunosupresores como la ciclosporina A.

La litotricia extracorpórea por ondas de choque (LEOC) es el tratamiento de elección en aquellos cálculos no complicados, situados en el riñón, menores de $2,5 \mathrm{~cm}$. y aquellos situados en el uréter, menores de $1,5 \mathrm{~cm}$., siempre y cuando no exista obstrucción distal al cálculo. Debido a la localización anterior del injerto, el paciente debe ser colocado en decúbito prono. En algunas series se han alcanzado tasas de éxito del $80 \%^{1}$, siendo necesarias varias sesiones, sin un mayor número de complicaciones que en los riñones nativos.

La ureterorrenoscopia presenta la dificultad de acceder al uréter reimplantado, debido a su posición y angulación.
El acceso percutáneo estaría indicado, cuando fracasan los tratamientos previos o existen alteraciones anatómicas (estenosis de la vía excretora). La localización superficial del riñón trasplantado facilita este abordaje, que fue utilizado por vez primera para extraer un cálculo en $1982^{7}$. Se han atribuido dos complicaciones a este procedimiento quirúrgico, la fístula urinaria y el urinoma perirrenal, después de la retirada del catéter, debido al retraso en la cicatrización secundario al tratamiento con esteroides ${ }^{3,7}$.

Desde que Schweizer y cols. comunicaron en 1977 la primera pielolitotomía abierta en un riñón trasplantado ${ }^{3}$, las indicaciones de la cirugía abierta de la litiasis del injerto renal ha quedado reservada a aquellos pacientes con cálculos de gran tamaño y difícil acceso o con patologías asociadas que requieren tratamiento asociado.

Podemos concluir que el tratamiento percutáneo de la litiasis en el riñón trasplantado, en aquellos casos seleccionados, representa una opción mínimamente invasiva con escasa repercusión sobre la función del injerto ${ }^{8}$ y un excelente resultado terapéutico.

\section{REFERENCIAS}

1. BORONAT TORMO F, PONTONES MORENO JL, BROSETA RICO E, OLIVER AMORÓS F, BUDIA ALBA A, JIMÉNEZ CRUZ JF.: Tratamiento de la litiasis renal cálcica. LEOC, NLP, cirugía abierta. Arch Esp Urol 2001; 54: 909-925.

2. HARPER JM, SAMUELL CT, HALLSON PC, WOOD SM, MANSELL MA.: Risk factors for calculus formation in patients with renal transplants. Br J Urol 1994; 74: 147-150.

3. RODRIGO ALIAGA M, MORERA MARTÍNEZ J, LÓPEZ ALCINA E, BROSETA RICO E, OLIVER AMORÓS F, BORONAT TORMO F, SÁNCHEZ PLUMED J, JIMÉNEZ CRUZ JF: Litiasis en el riñón trasplantado: posibilidades terapéuticas. Arch Esp Urol 1996; 49: $1.063-1.070$.

4. RUBIO BRIONES J, CHECHILE TONIOLO G, PARADA MORENO R, MINIÑO PIMENTEL L, GARCÍA PENIT J, CAPARRÓS SARIOL J, ROUSAUD BARÓN A.: Litiasis en trasplante renal. Actas Urol Esp 1995; 19: 561-565.

5. MONTAYNE G, JINDAL SL, IRVINE AH et al.: Calculus formation in renal transplant patients. J Urol 1984; 132: 448.

6. LERUT J, LERUT T, GRUWEZ JA et al.: Case profile: donor graft lithiasis. Unusual complication of renal transplantation. Urology 1979; 14: 627.

7. CALDWELL TC, BURNS JC.: Current operative management of urinary calculi after renal transplantation. J Urol 1988; 140: 1.360-1.363.

8. LANCINA JA, GARCÍA BUITRÓN JM, BERMÚDEZJD, ÁLVAREZ CASTELO L, DUARTE NOVO J, SÁNCHEZ MERINO JM, RUBIAL MOLDES M, GONZÁLEZ MARTÍN M.: Litiasis urinaria en riñón trasplantado. Arch Esp Urol 1997; 50: 141-150.

Dr. F. Ramón de Fata Chillón

Servicio de Urología. Hospital Universitario La Paz Po de la Castellana, 261 - 28046 Madrid

(Trabajo recibido el 20 febrero de 2002) 